\title{
Preface to the EAWE Portuguese version: A case for a new era of phenomenological psychopathology in psychiatry and clinical psychology
}

Elizabeth Pienkos ${ }^{1}$

Guilherme Messas²

This special issue presents a Portuguese translation of the EAWE: Examination of Anomalous World Experience (Sass et al., this issue; original publication Sass et al., 2017). The EAWE is a semi-structured, phenomenologically-oriented interview designed to elicit descriptions of changes in the lived world, particularly those that may be more commonly found in schizophrenia spectrum disorders (though it may also be used to study disorders outside the schizophrenia spectrum). The EAWE represents an important trend in psychiatric research and practice: a growing appreciation for the role of subjectivity in understanding, explaining (Sass, 2010), classifying and treating psychiatric disorders, especially complex and heterogeneous disorders such as schizophrenia. The EAWE's translation into Portuguese reflects several equally important developments. The ability to collect detailed information about the experience of schizophrenia and other psychiatric conditions among Portuguesespeaking participants will make it possible to study the phenomenological characteristics of schizophrenia outside of Anglophone cultures, providing important data about the intersections of individual, interpersonal, and cultural experiences in the development of the disorder. In addition, it occurs at a time of renewed energy and collaboration in phenomenological psychopathology, particularly in Brazil.

The EAWE considers six crucial domains of world experience, all of which tend to be altered in schizophrenia: Space and objects, Time and events, Other persons, Language, Atmosphere, and Existential orientation. (Further discussion of the forms of experience in schizophrenia of each of these domains can be found in the six ancillary articles to the EAWE in the special issue of Psychopathology [Sass, Pienkos, \& Fuchs, 2017] devoted to the interview.) It was developed to complement the EASE: Examination of Anomalous Self

\footnotetext{
${ }^{1}$ Psychology Department, Clarkson University, Potsdam, NY, USA

E-mail: epienkos@ clarkson.edu.

${ }^{2}$ Faculdade de Ciências Médicas da Santa Casa de São Paulo, São Paulo, Brazil
}

Psicopatologia Fenomenológica Contemporânea - EAWE, 2018; 7(2):1-9 
Experience (Parnas et al., 2005), which emphasizes changes in selfhood that accompany schizophrenia, which have since been shown to be more numerous in schizophrenia and groups at risk for developing schizophrenia compared to other psychiatric groups (e.g. Nordgaard \& Parnas, 2014; Raballo, Saebye, \& Parnas, 2011). As has been emphasized by phenomenological philosophers, including Husserl, Merleau-Ponty and Heidegger, experiences of the self and the world are inextricably interwoven. This involves the concept of intentionality: that consciousness is always object- or world-directed, is always conscious of something. Alterations in consciousness will therefore always involve alterations in the awareness of the things they are conscious of. In the words of Merleau-Ponty (1945) "subject and object" are "two abstract moments of a unique structure, namely, presence" (p. 500). As Madeira et al. (this issue) argue, awareness of the ways one encounters objects, people, and events in the world is crucial to understanding the nature of psychiatric disorders and their impact on individuals.

The EAWE may elicit rich qualitative data, and it may also be used to determine the number and types of alterations experienced by an individual. The interview has been structured to enable clear and consistent administration, and it has been demonstrated to have strong interrater reliability and internal consistency (Conerty et al., 2017). Interviewing requires that the interviewer bracket his or her own assumptions and explanatory models about experience and, instead, facilitate a clear description of the ways the world appears to the interviewee. Use of the EAWE also requires a background familiarity with phenomenological psychopathology, which helps the interviewer to be sensitive to the potential nuances and interrelated features of the experiences being described. In addition to its use as an interview tool, the EAWE is designed to illustrate the range of anomalous experiences that may occur in schizophrenia, to sensitize researchers and clinicians to features that are not commonly included in contemporary diagnostic and assessment methods, and to facilitate the discussion of these aspects with those who suffer from schizophrenia.

As Pienkos and Sass (this issue) note, this structure allows interviewees to clearly describe the nature of their experiences without the artificial limitations imposed by circumscribed symptom definitions. This is especially important for schizophrenia, where 
many of the experiential changes associated with the disorder often seem to defy description. It may also be essential in cross-cultural research on the disorder, as a semi-structured approach is more likely to permit the expression of culturally-specific features of experience. Comparison of groups from diverse cultures may be able to further clarify the essential features of the disorder and the ways these features are embedded in the individual situation, which includes social and cultural norms, traditions, and forms of expression. Or as Messas and Tamelini (in press) state, "we can no longer content ourselves with a general interpretation of a pathological experience... but must see how this generality presents itself in particularities." Such phenomenologically- and culturally-informed research will undoubtedly contribute to the goals of contemporary psychiatry and psychology of respecting and incorporating individual and group differences, without sacrificing reliability or scientific rigor.

The publications of the EAWE and this translation occur at an important time for determining a new role for phenomenology in contemporary psychiatry and clinical psychology. As described by Madeira et al. (this issue), these disciplines continue to promote their legitimacy through identification with the natural sciences, which often means enhancing reliability and operationalizing psychological variables to facilitate their study as scientific objects. Of course, the ability to design controlled studies and ensure that researchers and clinicians can reach agreement about the complex behaviors and processes they study is essential to the advancement of these fields. However, standardized interviews and self-report measures, ostensibly developed to minimize variability in administration, leave little room for the variability inherent in the interviewee, potentially limiting the expression of important features and phenomena, both cultural and individual. Furthermore, they make assumptions about which features are important to the diagnosis and treatment of psychiatric disorders (Pienkos at al., in press); because research often relies on the use of these instruments, researchers limit themselves to collecting data that is constrained by these assumptions.

The approach of phenomenological psychopathology provides a crucial corrective to these trends. Of course, it is not a novel approach, and it has been developed and applied to numerous forms of mental disorder for over 100 years, starting with the publication of Karl 
Jaspers's General Psychopathology (1963) in 1913. But recent developments in its methods make phenomenology increasingly accessible and useful to psychiatry and psychopathology. The EAWE: Examination of Anomalous World Experience, with its systematic and flexible approach to phenomenological inquiry, reflects some of these developments. The EAWE can also empower the scientific community with a new conceptual and pragmatic instrument for addressing two fundamental challenges of contemporary psychiatry and clinical psychology, though we can only sketch these topics here. The first of these is concerned with the nature of psychopathological knowledge, that is, how best to access psychological or subjective reality; the second, with the influence of this knowledge on clinical practice.

Perhaps the most obvious value of the EAWE is its restoration of the first-person epistemological perspective as the cornerstone of the understanding and assessment of the patient's psychopathological experiences (Leal \& Serpa Jr., 2013). There is a long history of debate in phenomenological psychopathology regarding the best way to access the nature of subjective experience, i.e. via the "first-person" or "second-person" perspective (Galbusera $\&$ Fellin, 2014). Here, the first-person approach is understood as the self-descriptions of a subject or patient, relying either on a spontaneous narrative or on her self-descriptions collected by an open (van der Praag, 1992) or a semi-structured interview (like the EAWE). The second-person approach may be understood as the interpersonal elaboration or interpretation of this first-person material, based either on a hermeneutic-linguistic interpretation (Fuchs, 2010) or on a comprehensive analysis of the simultaneous, cooccurring, or resonant experiences of the patient and the psychopathologist (Minkowski, 1995; Kimura, 2000; Messas \& Fukuda, 2018). The main attitude of the researcher in the first-person approach follows the classic Jaspersian proposition of the researcher's empathic grasping of the lived world of the patient, by putting herself in the place of the patient (Hineinversetzen) (Jaspers, 1966). On the other hand, the basic attitude of the second-person approach is, in the elegant words of Depraz et al. (2011), “... répresentée par un tuteur ou un guide qui posséde un degree plus avéré d'apprentissage ou de competence dans un domaine..." (“....represented by a tutor or a guide who has an advanced degree of knowledge or competence in a specific domain..."; authors' translation) (p. 122); in this case, the domain of existence. It is evident that there are not clear boundaries between the first-person and 
second-person approaches, and that they need each other for a comprehensive access to the patient's experiences. ${ }^{3}$ Though a discussion of this debate is beyond the scope of this short preface, its consequences are by no means trivial, as the positions of this controversy involve the very definition of the ontological status of existence, i.e. the definition of the psychopathological object either as an isolated or solipsistic form of disturbed experience, or as an interpersonally constituted one. (Oulis 2008; Messas \& Tamelini, in press; Nordgaard et al., 2012).

One of the most noteworthy features of EAWE is its ability to both escape and further this debate, by allowing a kind of "double access" to human experience: the interview can be used for a pure investigation of subjectivity (representing a first-person approach), and, we would suggest, it may also be used for the investigation of correspondence or attunement of the experiences of the patient and the experiences of the researcher (the second-person approach). (Of course, the EAWE may also be used as a more traditional instrument of the third-person perspective, recording and assessing various subjective complaints and disturbances using reliable, semi-systematized methods.) This second use of EAWE may correct the unfortunate underdevelopment of one of the most relevant insights of the phenomenological tradition, the acknowledgement of the importance of the reactions of the psychopathologist as a fundamental part of the psychopathological investigation (Minkowski, 1995; Rümke, 1990). For example, the information collected in the "Other persons" domain might be correlated with the impressions of the researcher during the interview, and potentially used for further discussion with the interviewee. To our knowledge, this is the first phenomenological-based instrument that permits this simultaneous consideration of both perspectives on subjectivity.

The second contribution of the EAWE to contemporary psychiatry and clinical psychology is its application to clinical decision-making, which is relevant both to individual patients and to larger research agendas. Decision-making relies on the individual complexities of subjective experience. This uniqueness of human experience, specific to each individual, makes the development of instruments for capturing the singular features of individual patients a new frontier in psychiatry and clinical psychology (Messas et al., 2017).

\footnotetext{
${ }^{3}$ For a fuller discussion of the topic, we refer to Gallagher \& Zahavi, 2008.
} 
The EAWE, with its detailed analysis of individual experiences, highlights essential but often overlooked features of experience, which may be necessary for a phenomenologicallyinformed, individualized approach to conceptualization and clinical decision-making. In addition, as psychiatry and related disciplines strive to integrate the influences of both biology and biography in their understanding of human life, phenomenology may be able to offer important clues and directions for more sophisticated approaches to research, particularly related to etiology and treatment (Messas, 2010; Sass et al., 2018). The EAWE, by allowing greater access to the totality of individual experience, may improve both idiographic decision-making and the nomothetic understanding of complex disorders. It may help, for instance, to elucidate the relationship(s) between psychotic disorders and substance abuse, contributing to the (so far) largely unexplored agenda of the lived experiences of drug misuse and psychosis (Messas, 2016).

Finally, some words about several new trends in phenomenological psychology in Brazil. Although phenomenology has a decades-long tradition in Brazil (Andrade \& Holanda, 2010), and there are many societies dedicated to this theme informed by several distinct tendencies in phenomenology (such as Sociedade Brasileira de Psicopatologia Fenômeno-Estrutural (SBPFE, the sponsor of this journal), it is only this year that a unifying federation society has been founded: the Associação Brasileira de Psicologia Fenomenológica (ABRAPFE). This recent confluence of the distinct - and often isolated phenomenology tendencies in Brazil strengthens the relevance of phenomenological psychiatry and psychology in the society, as well as in the country. This trend not only makes the introduction of the EAWE welcome, but also necessary to improve the dialogue between the various phenomenological currents, and also between phenomenology and contemporary culture in Brazil. Perhaps more importantly, given the still largely insufficient use of phenomenological theory in clinical practice (Dourado et al., 2016), the EAWE should be welcomed as a token of a new era of phenomenologically-informed approach to scientific discovery and clinical practice in Brazil.

We hope that this era will be marked by a closer dialogue between phenomenology and the everyday language and values of society, one that includes and prioritizes the classic phenomenological respect for a deep understanding of the main themes of human existence. 


\section{References}

Andrade, C. C., \& Holanda, A. F. (2010) Apontamentos sobre pesquisa qualitativa e pesquisa empírico-fenomenológica. Estudos de Psicologia, Campinas v. 27, n. 2, 259-268, abr./jun. 2010.

Conerty, J., Skodlar, B., Pienkos, E., Byrom, G., \& Sass, L. (2017). EAWE: Examination of Anomalous World Experience: A report on reliability. Psychopathology, 50, 55-59.

Dourado, C., \& Moreira V., Melo A. Revisão sistemática de literatura sobre a Psicopatologia Fenomenológica no Brasil [Systematic review of literature on the Phenomenological Psychopathology in Brazil]. Psicopatologia Fenomenológica Contemporânea, 2016; 5(2): pp.111-144.

Depraz, N., Varela, F. J., \& Vermersch, P. À l'épreuve de l'expérience. Pour une pratique phénoménologique. Bucarest: ZETA Books.

Fuchs, T. (2010). Subjectivity and intersubjectivity in psychiatric diagnosis. Psychopathology, Basel, v. 43, n. 4, 268-74, jun. 2010.

Galbusera, L., \& Fellin, L. The intersubjective endeavor of psychopathology research: methodological reflections on a second-person perspective approach. Front Psychol, Lausanne, v. 5, article 1150, 1-14, oct. 2014.

Gallagher, S., \& Zahavi, D. (2008). The phenomenological mind: An introduction to philosophy of mind and cognitive science. New York: Routledge.

Jaspers, K. (1963). General Psychopathology. Chicago: University of Chicago Press.

. The phenomenological approach in psychopathology. Brit J Psychiat, London, v. 114, n. 516, 1313-1323, nov. 1968.

Kimura, B. (2000). L'entre. Une approche phenomelogique de la schizophrenie. Grenoble: Jérôme Millon.

Leal, E. M.; Serpa JR, O. D. (2013). Acesso à experiência em primeira pessoa na pesquisa em Saúde Mental. Ciênc. saúde coletiva, Rio de Janeiro, v. 18, n. 10, 2939-48, jun. 2013

Madeira, L., Filipe, T., Carneiro, T., \& Figueira, M.L. (2018). The loss of nosological validity: Why and how should we consider disturbances of subjective world

Psicopatologia Fenomenológica Contemporânea - EAWE, 2018; 7(2):1-9 
experience? Psicopatologia Fenomenológica Contemporânea XXX.

Messas, G. (2010). A Phenomenological Contribution to the Approach of Biological Psychiatry. Journal of Phenomenological Psychology 41, 180-200.

. (2016). The association between substance use/misuse and psychosis: A phenomenological viewpoint. Comprendre, 25-26, 251-266.

Messas, G., Fulford, K. W., \& Stanghellini, G. The contribution of human sciences to the challenges of contemporary psychiatry. Trends Psychiatry Psychother, Porto Alegre, v. 39, n. 4, 229-231, oct./dec. 2017.

Messas, G., \& Fukuda, L. O diagnóstico psicopatológico fenomenológico da perspectiva Dialético-essencialista. Revista Pesquisa Qualitativa. São Paulo (SP), v.6, n.11, p. 160-191, ago. 2018.

Messas, G. P.; Tamelini, M. G. The Pragmatic Value of Notions of Dialectics and Essence in Phenomenological Psychiatry and Psychopathology. Thaumàzein - Rivista di Filosofia, Verona, in press.

Minkowski, E. (1995). Le temps vécu. Paris: Presses Universitaires de France.

Nordgaard, J.; Sass, L.; Parnas, J. The psychiatric interview: validity, structure, and subjectivity. Eur Arch Psychiatry Clin Neurosci, Berlin/Heidelberg, v.263, n.4, p. 353-364, jun. 2013.

Nordgaard, J., \& Parnas, J. (2014). Self-disorders and the schizophrenia spectrum: A study of 100 first hospital admissions. Schizophrenia Bulletin, 30, 1300-1307.

Oulis, P. Ontological assumptions of psychiatric taxonomy: main rival positions and their critical assessment. Psychopathology, Basel, v. 41, n. 3, p. 135-40, jan. 2008

Parnas, J., Moller, P., Kircher, T., Thalbitzer, J., Jansson, L., Handest, P., \& Zahavi, D. (2005). EASE: Examination of Anomalous Self-Experience. Psychopathology, 38, 236-258

Pienkos, E., Giersch, A., Hansen, M., Humpston, C., McCarthy-Jones, S., Mishara, A., Nelson, B., Park, S. Sharma, R., Thomas, N. \& Rosen, C. Hallucinations beyond voices: A conceptual review of the phenomenology of altered perception in psychosis. Schizophrenia Bulletin.

Psicopatologia Fenomenológica Contemporânea - EAWE, 2018; 7(2):1-9 
Pienkos, E., \& Sass., L. (2018). Schizophrenia, language, and the phenomenological interview. Psicopatologia Fenomenológica Contemporânea XXX.

Raballo, A., Saebye, D., \& Parnas, J. (2011). Looking at the schizophrenia spectrum through the prism of self-disorders: An empirical study. Schizophrenia Bulletin, 37(2), 344351.

Rümke, H. (1990). The nuclear symptoms of schizophrenia and the praecox feeling. History of Psychiatry, 1, 331-341.

Sass, L. (2010). Phenomenology as description and as explanation: The case of schizophrenia. In S. Gallagher \& D. Schmicking (Eds.), Handbook of phenomenology and cognitive science (pp. 635-654). New York: Springer.

Sass, L., Borda, J., Madeira, L., Pienkos, E., \& Nelson, B. (2018). Varieties of self-disorders: A bio-pheno-social model of schizophrenia. Schizophrenia Bulletin, 44, 720-727.

Sass, L., Pienkos, E., \& Fuchs, T. (Eds.). 2017. Other worlds: The Examination of Anomalous World Experience (EAWE) [Special issue]. Psychopathology, 50(1).

Sass, L., Pienkos, E., Skodlar, B., Stanghellini, G., Fuchs, T., Parnas, J., \& Jones, N. (2018). EAWE: Examination of anomalous world experience. (L. Madeira, A. Spremberg, G. Queiroz, \& J. Eira, trans.). Psicopatologia Fenomenológica Contemporânea XXX.

Sass, L., Pienkos, E., Skodlar, B., Stanghellini, G., Fuchs, T., Parnas, J., \& Jones, N. (2017). EAWE: Examination of anomalous world experience. Psychopathology, 50(1), 1054.

Psicopatologia Fenomenológica Contemporânea - EAWE, 2018; 7(2):1-9 